\title{
Precise pointing knowledge for SCIAMACHY solar occultation measurements
}

\author{
K. Bramstedt ${ }^{1}$, S. Noël ${ }^{1}$, H. Bovensmann ${ }^{1}$, M. Gottwald ${ }^{2}$, and J. P. Burrows ${ }^{1}$ \\ ${ }^{1}$ Institute of Environmental Physics (IUP), University of Bremen, Otto-Hahn-Allee 1, 28359 Bremen, Germany \\ ${ }^{2}$ German Aerospace Center, Remote Sensing Technology Institute, Münchner Str. 20, 82234 Wessling, Germany \\ Correspondence to: K. Bramstedt (klaus.bramstedt@iup.physik.uni-bremen.de)
}

Received: 30 April 2012 - Published in Atmos. Meas. Tech. Discuss.: 31 May 2012

Revised: 17 October 2012 - Accepted: 1 November 2012 - Published: 27 November 2012

\begin{abstract}
We present a method to precisely determine the viewing direction for solar occultation instruments from scans over the solar disk. Basic idea is the fit of the maximum intensity during the scan, which corresponds to the center of the solar disk in the scanning direction. We apply this method to the solar occultation measurements of the satellite instrument SCIAMACHY, which scans the Sun in elevation direction. The achieved mean precision is $0.46 \mathrm{mdeg}$, which corresponds to an tangent height error of about $26 \mathrm{~m}$ for individual occultation sequences. The deviation of the derived elevation angle from the geolocation information given along with the product has a seasonal cycle with an amplitude of $2.26 \mathrm{mdeg}$, which is in tangent height an amplitude of about $127 \mathrm{~m}$. The mean elevation angle offset is $-4.41 \mathrm{mdeg}$ ( $249 \mathrm{~m})$. SCIAMACHY's sun follower device controls the azimuth viewing direction during the occultation measurements. The derived mean azimuth direction has an standard error of $0.65 \mathrm{mdeg}$, which is about $36 \mathrm{~m}$ in horizontal direction at the tangent point. We observe also a seasonal cycle of the azimuth mispointing with an amplitude of $2.3 \mathrm{mdeg}$, which is slightly increasing with time. The almost constant mean offset is $88 \mathrm{mdeg}$, which is about $5.0 \mathrm{~km}$ horizontal offset at the tangent point.
\end{abstract}

\section{Introduction}

For global observations of atmospheric constituents, satellite measurements are a key component. Passive remote sensing instruments observing the atmosphere use basically three types of geometries: nadir, limb and occultation. The nadir geometry primarily provides column information of trace gases and aerosol, albedo and cloud properties. Observations in limb or occultation geometry can provide height resolved information. A critical point in the latter two is always the pointing knowledge, i.e. the precise knowledge of the viewing direction and with that the observed tangent height.

SCIAMACHY (SCanning Imaging Absorption spectroMeter for Atmospheric CHartographY) is a passive remote sensing moderate-resolution imaging UV-Vis-NIR spectrometer on board the European Space Agency (ESA) Environmental Satellite (Envisat). Envisat was launched in March 2002 from Kourou, French Guiana to a sun synchronous orbit with an equator crossing time in the descending node of 10:00 a.m. LT. In April 2012, the contact to Envisat was lost. In May 2012, ESA declared the end of Envisat's operational in-orbit phase. The instrument observed the Earth atmosphere in nadir, limb and solar/lunar occultation geometries and provided column and profile information of atmospheric trace gases of relevance to ozone chemistry, air pollution, and climate monitoring issues (Bovensmann et al., 1999; Gottwald and Bovensmann, 2011).

SCIAMACHY performs its solar occultation measurement in the Northern Hemisphere, when the Sun rises due to the orbital motion of the platform. On the day side of the orbit, alternating limb and nadir measurements are performed in such way that the same air-masses are probed first by limb and a few minutes later in nadir. If the Moon is with a phase larger than 0.5 in the field of view, lunar occultation is performed at moonrise in the Southern Hemisphere. During eclipse, dark current measurements are performed. Since 2008, also limb mesospheric states are executed during eclipse. Various additional monitoring measurements are performed in the daily, weekly and monthly calibration. 
SCIAMACHY is a passive imaging spectrometer. The scan mirror system comprises the elevation scanner and the azimuth scanner. The spectrometer entrance slit defines an instantaneous field of view (IFOV) of $1.8 \mathrm{deg} \times 0.045 \mathrm{deg}$. In case of solar occultation, a small aperture reduces the amount of incoming light, limiting the field of view to $0.72 \mathrm{deg} \times 0.045 \mathrm{deg}$. The spectrometer has eight detector modules with 1024 detector pixels each, covering the ultraviolet, visible and near-infrared spectral range. Seven broadband detectors, the polarization measurement devices (PMDs), are used to determine the polarization state of the incoming light. PMD 1-6 measure the light polarized perpendicular to SCIAMACHY's optical plane. PMD 7 is sensitive to the $45 \mathrm{deg}$ component in the visible spectral range. The PMDs are sampling devices, whereas the spectral detectors are integrating devices. The spectral characteristics of all detectors are listed in Table 1.

The original requirements of pointing performance are not very strict: the absolute knowledge error in elevation shall be less than $\pm 0.075 \mathrm{deg}$, the absolute altitude shall be known within $\pm 4.3 \mathrm{~km}$. The relative i.e. pointing error shall be less than \pm 0.028 deg, i.e. the tangent height offsets of individual measurements may vary within $\pm 1.6 \mathrm{~km}$. The requirements in azimuth direction have even larger values. Before launch, the pointing error budget of SCIAMACHY was estimated by Schwab (1999). The overall pointing error budget considers the pointing performance of the platform and of the instrument. This budget is dominated by the spacecraft, because large error budgets for the orbit state vector accuracy and the attenuation especially around the pitch axis were assumed. The error budget for SCIAMACHY only is mainly related to possible thermal distortions. Two numbers are given in the error budget: the relative pointing error is the precision of the pointing, the absolute pointing error is the accuracy, i.e. the precision plus a possible bias.

The absolute pointing knowledge error budget in elevation/azimuth is $0.045 \mathrm{deg}(2.5 \mathrm{~km}) / 0.058 \mathrm{deg}(3.3 \mathrm{~km})$. SCIAMACHY's part of the budget is $0.019 \mathrm{deg}$ for both directions. The budget of the relative pointing error in elevation is $0.016 \mathrm{deg}$, with a budget of $0.008 \mathrm{deg}$ for SCIAMACHY alone. No budget for the relative azimuth pointing error is given. If SCIAMACHY's sun follower device (SFD) is used, the absolute pointing error of the platform is estimated with $0.012 \mathrm{deg}(0.020 \mathrm{deg})$ in elevation (azimuth). SCIAMACHY's part of the budget is $0.005 \mathrm{deg}(0.010 \mathrm{deg})$.

Deriving tangent height information from limb measurements is possible with the Tangent height Retrieval by UV-B Exploitation (TRUE) method, which analyzes the so-called knee in the shape of UV-B limb radiance profiles (Kaiser et al., 2004). For the tropical region with its homogenous ozone distribution, the uncertainty of the knee method is estimated to be $300 \mathrm{~m}$ or less (von Savigny et al., 2005).

In the early phase of the mission, large tangent height anomalies were observed with this method with a seasonal variation with an amplitude of $800 \mathrm{~m}$ (von Savigny et al.,
Table 1. Spectral characteristics of the science channels and the PMDs. The name of the PMDs is used in many SCIAMACHY documents, in this paper the number is used. The PMD wavelength range is defined such that it contains $80 \%$ of the signal, the range of the science channels is the total range.

\begin{tabular}{rrrllr}
\hline Ch. & $\begin{array}{r}\text { Wavelength } \\
\text { range [nm] }\end{array}$ & $\begin{array}{c}\text { Resolution } \\
{[\mathrm{nm}]}\end{array}$ & $\begin{array}{l}\text { PMD } \\
\text { name }\end{array}$ & $\begin{array}{l}\text { PMD } \\
\text { no. }\end{array}$ & $\begin{array}{r}\text { Wavelength } \\
\text { range [nm] }\end{array}$ \\
\hline 1 & $212-334$ & 0.24 & - & - & - \\
2 & $300-412$ & 0.26 & A & 1 & $310-365$ \\
3 & $383-628$ & 0.44 & B & 2 & $455-515$ \\
4 & $595-812$ & 0.48 & C & 3 & $610-690$ \\
5 & $773-1063$ & 0.54 & D/45 & $4 / 7$ & $800-900$ \\
6 & $971-1773$ & 1.48 & E & 5 & $1500-1635$ \\
7 & $1934-2044$ & 0.22 & - & - & - \\
8 & $2259-2386$ & 0.26 & F & 6 & $2280-2400$ \\
\hline
\end{tabular}

2005). Similar results are found by other instruments on Envisat: ASAR, GOMOS, and MIPAS (Bargellini et al., 2005). A detailed investigation by ESA lead to an update of the onboard attitude control software by an optimization of the onboard orbit model in December 2003 (Bargellini et al., 2005), which lead to a major improvement of SCIAMACHY's pointing performance. The remaining seasonal cycle derived with TRUE had an amplitude of about $220 \mathrm{~m}$ and a mean offset of about $1 \mathrm{~km}$. Investigation of monitoring measurements improved the knowledge about SCIAMACHY's misalignment (Gottwald et al., 2007), which considerably reduced the mean offset. The current status of the pointing knowledge is described in Gottwald et al. (2010), which states the overall accuracy reached so far with $2-3$ mdeg in elevation, corresponding to about $110-170 \mathrm{~m}$ in tangent height.

After the update of Envisat's on-board software, ESA also introduced an additional auxiliary file for the operational calculation of the geolocation, representing the best knowledge of Envisat's attitude also for the early orbits of the mission (see AUX-FRAs in Sect. 3.2). Therefore the actual pointing knowledge is available for the whole mission in the recent (reprocessed) product version 7.04 of Level $1 b$.

In this paper, we describe a method to derive precise tangent heights from the scans over the solar disk for the solar occultation measurements. We will further improve the tangent height knowledge to an uncertainty of about $26 \mathrm{~m}$ in altitude for the solar occultation measurements. By utilizing SCIAMACHY's SFD, the uncertainty in the horizontal direction will be reduced to about $36 \mathrm{~m}$.

\section{SCIAMACHY's solar occultation measurement}

SCIAMACHY performs solar occultation measurements every orbit between $49^{\circ} \mathrm{N}$ and $69^{\circ} \mathrm{N}$ depending on season (Fig. 1). From the instruments point of view, the Sun rises above the horizon, but the local time at the tangent point corresponds to a sunset event. The scan sequence is illustrated 


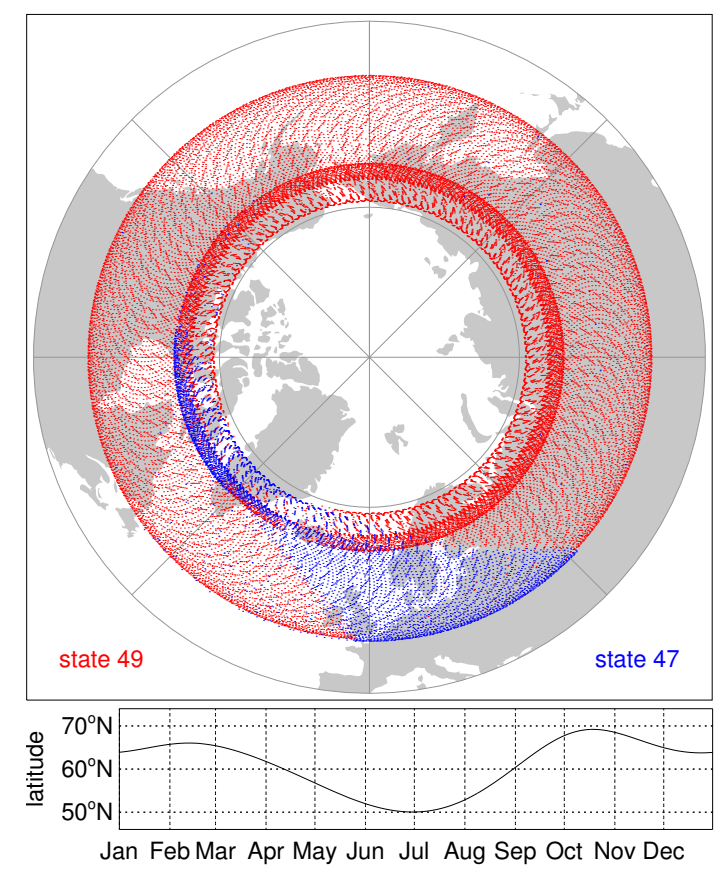

Fig. 1. Geographical distribution of SCIAMACHY solar occultation events. Top panel: all tangent points for the years 2003 to 2010 are plotted. Red dots are the nominal solar occultation measurements (state 49), blue dots are the shortened measurements (state 7). Bottom panel: the tangent points latitude over the year.

in Fig. 2: in an initial phase, SCIAMACHY scans around $17.2 \mathrm{~km}$ above the horizon, waiting for the Sun to appear. When the Sun's geometrical altitude exceeds $17.2 \mathrm{~km}$, the instrument follows (continuously scanning in vertical direction) the rising Sun up to an altitude of about $290 \mathrm{~km}$. For two orbits per day, scanning the Sun stops at about $110 \mathrm{~km}$, followed by two seconds pointing to the brightest region of the Sun, using the instrument's SFD. The remaining time is used for solar measurements of the daily calibration.

A set of so-called states define SCIAMACHY's measurements along the orbit. A state comprises a functional sequence of measurements. Each state definition has a unique number. The long solar occultation sequence sketched in Fig. 2 has the state number 49 , the short sequence used for 2 of 14 orbits per day has the state number 47 .

The IFOV in horizontal direction with 0.72 deg exceeds the diameter of the solar disk ( 0.525 to $0.542 \mathrm{deg}$, depending on season). In vertical direction, a sweep of $0.72 \mathrm{deg}$ in two seconds is performed. The IFOV in vertical direction is $0.045 \mathrm{deg}$ corresponding to an altitude range of about $2.5 \mathrm{~km}$ at the tangent point. With measurements performed every $125 \mathrm{~ms}$ (channel 3-6 and 8), 16 spectra are taken during one sweep of $0.72 \mathrm{deg}$ over the solar disk. The disk is completely covered, as illustrated in Fig. 3.

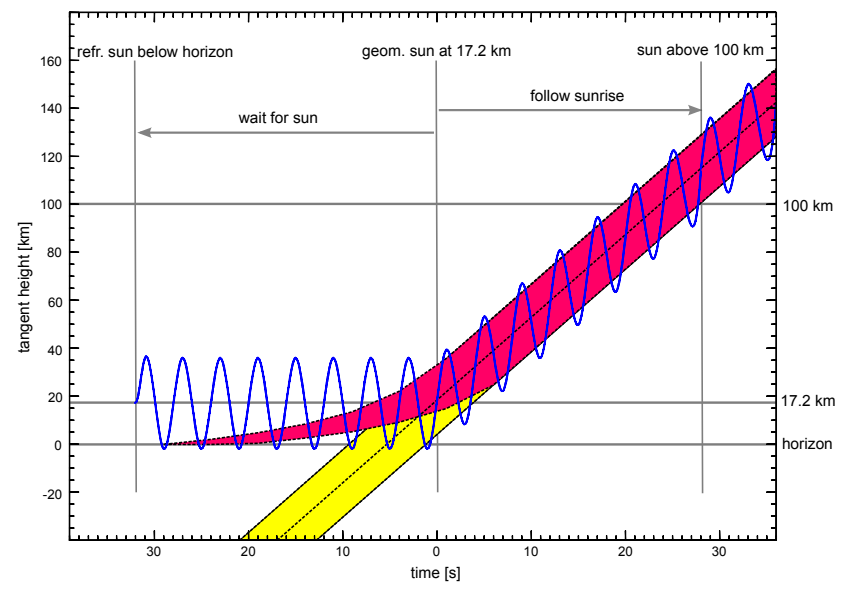

Fig. 2. Outline of the SCIAMACHY solar occultation measurement sequence. The yellow area indicates the geometrical position of the solar disk. The red area shows the visibility of the Sun, changed from the geometrical position by refraction in the Earth's atmosphere. The sinusoidal line gives the viewing direction of the instrument during the sequence, which is continued up to an altitude of $290 \mathrm{~km}$ for the nominal scan (state 49). The shortened measurement stops scanning at $110 \mathrm{~km}$, followed by $2 \mathrm{~s}$ pointing towards the Sun, adjusted by SCIAMACHY's sun follower device (state 47).

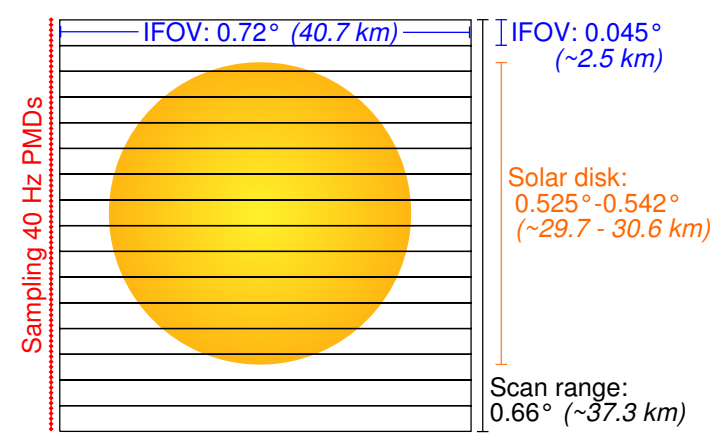

Fig. 3. SCIAMACHYs instantaneous field of view (IFOV) compared to the solar disk, detailed explanation is given in the text. The red points on the left side indicate the sampling of the PMDs during one scan.

\section{Elevation angle offset}

We use the scans over the solar disk above the atmosphere to determine the elevation angle of the Sun from Envisat's point of view. The elevation angle determines the tangent height for the viewing direction, but our basic result will be the mispointing in elevation direction. From the scan, the time of the maximum intensity of the solar irradiance is fitted. The viewing direction at this point in time is the measured elevation direction of the Sun. Using the best knowledge of the attitude and position of the platform, we can calculate the elevation direction of the Sun with an appropriate software. This software is also used in the operational processing to calculate the geolocation of the measurement from the same 
input. The difference between the measured and the calculated elevation direction of the Sun gives the mispointing of the instrument in elevation: the elevation angle offset (EAO). The detailed implementation of this basic idea is described in the following paragraphs.

An EAO can be translated to a more intuitive tangent height offset. In principle, the conversion factor is altitude dependent, and this dependence also has a seasonal component. Nevertheless, an estimate of the tangent height offset is given at the right axis of most plots, using a mean conversion factor.

\subsection{The measured position of the Sun}

Neglecting the vertical extension of the FOV, the measured intensity on the solar disk is proportional to the length of the chord defined by the horizontal extent of SCIAMACHY's FOV (Fig. 4). By using the theorem of Pythagoras, we get

$I(t)=2 c \sqrt{r^{2}-\left(t-t_{\mathrm{cen}}\right)^{2}}$

with

$t_{\text {cen }}$ : center of the solar disk,

$t$ : vertical position of the FOV,

$r$ : radius of the Sun,

$c$ : scaling factor,

$I(t)$ : measured intensity.

The vertical direction is proportional to the time, because the scan is performed with constant angular velocity of the elevation mirror. Therefore, $t, t_{\mathrm{cen}}$ and $r$ can be expressed in (measurement) time coordinates. The maximum measured intensity is then given by

$I_{\max }=2 c r$.

The parameters $r, c$, and $t_{\text {cen }}$ are fitted with a non-linear least square algorithm (Levenberg-Marquard; Lourakis, 2009) to the measurement points during the scan over the solar disk. $t_{\text {cen }}$ then gives the precise time, when the FOV is centered over the solar disk.

This approach has two main sources of errors. First, the field of view indeed has a vertical extension. Second, the solar disk is not a homogenous source of light. The main effect is the solar limb darkening. The brightness of the Sun is decreasing from the center to the edge of the Sun because of absorptions in the Sun's atmosphere. This effect is wavelength dependent, it is decreasing from UV towards longer wavelengths (Hestroffer and Magnan, 1998; Neckel, 2005). Both effects are increasing with the distance of the FOV from the center of the Sun.

To increase the numerical stability of the fit, the values of the fit are prepared as follows: the time is always given as seconds from the begin of the state, i.e. since the begin of the occultation measurement sequence. The intensity values are normalized to the maximum intensity of the state.

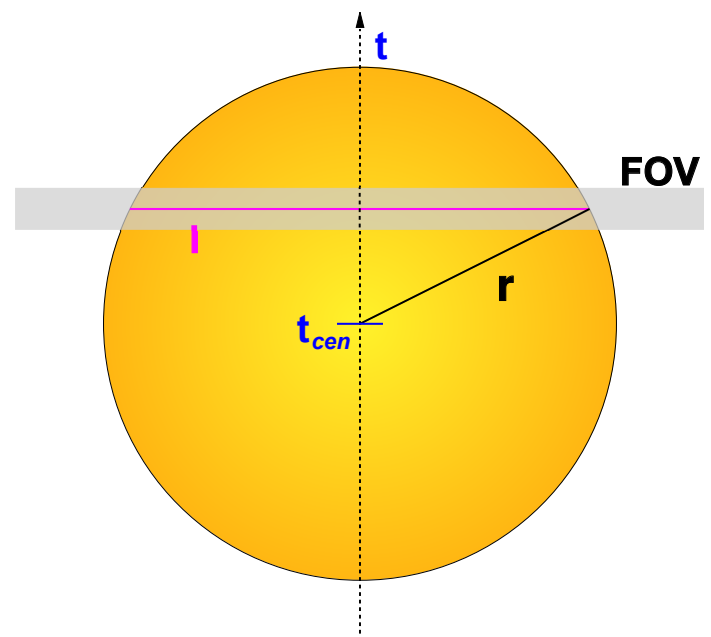

Fig. 4. Sketch for deriving the intensity function of a scan over the solar disk.

\subsection{The calculated position of the Sun}

All calculations necessary for orbit propagation and pointing issues for Envisat are combined in the Envisat-1 mission CFI (Customer Furbished Item) software (Muñoz, 2011), in the following named as CFI library. The software needs an orbit state vector with position and velocity of the spacecraft at a dedicated time, which then can be propagated along the orbit. Each SCIAMACHY product contains the orbit state vector at approximately the ascending node crossing (ANX) time. We use the orbit state vector from the SCIAMACHY Level $1 \mathrm{~b}$ product version 7.04. In addition to a detailed Earth model, the software includes a model of the main bodies in the solar system and the positions of the (brighter) stars. It also includes sophisticated routines to convert positions, velocities, and accelerations between the various coordinate systems related to the solar system, the Earth and the platform. The most relevant coordinate system (CS) for our purpose is the Satellite Relative Actual Reference CS (SRAR CS). This is a satellite fixed CS, using the (best available) attitude information for the platform and the known (fixed) mispointing angles. The positions of SCIAMACHY's elevation and azimuth mirrors are proportional to the elevation and azimuth angles in this coordinate system, i.e. the viewing direction of SCIAMACHY is defined only by the mirror positions in this CS (Alvarez, 1997).

To calculate the elevation and azimuth angle of the Sun in the SRAR CS, the attitude of the satellite must be known. The attitude information is determined using Envisat's gyroscopes, the Earth and the Sun sensor, and the three star sensors. Using the star sensors gives the most precise attitude information, this is Envisat's operational stellar yaw steering mode (SYSM). As a backup operational mode, the yaw steering mode (YSM) with limited attitude information is possible (Bargellini et al., 2005). 
With a delay of two to three days after the measurement, the FOS (Flight Operation Segment) Restituted Attitude Data File is available, the so-called AUX-FRAs (after the identification string in the file names). They improve the attitude knowledge by a detailed re-analysis of all available information in the FOS and can be applied within the CFI library to improve the propagation along the orbit.

Previous CFI libraries assumed an infinite speed of light. With version 5.9, the possibility to correct for the traveling time of light for some Sun related parameters was added, including the ones needed for this work. We checked this correction with calculations performed with the The JPL HORIZONS online solar system data and ephemeris computation service of the Solar System Dynamics (SSD) group of NASA's Jet Propulsion Laboratory (JPL) (Giorgini et al., 1996; Yeomans et al., 1996), and found a very good agreement between them with differences below 0.3 mdeg. All results presented here use the correction for the traveling time of light.

If the measured elevation or azimuth angle differs from the calculated one, the attitude information of the instrument is incorrect and the Satellite Relative Actual Reference CS is not exactly aligned with SCIAMACHY.

\subsection{Optimizing the fit}

As mentioned before, SCIAMACHY has two types of detectors (see Table 1). The science channels are integrating devices with a readout rate not larger than $16 \mathrm{~Hz}$. The PMDs are broadband sampling devices, which are read out with $40 \mathrm{~Hz}$. For operational purpose, they are regridded with a $32 \mathrm{~Hz}$ sampling, synchronized with the readings of the science channels. Here, we work with the raw $40 \mathrm{~Hz}$ signal to take advantage of the high sampling rate.

The light path to PMD 7 is essentially blocked by the small aperture in solar occultation. However, a small amount of stray light still reaches this detector, but the signal is too noisy to be used. PMD 6 also has quite noisy data. We performed fits for all 7 PMDs and compared the resulting fit errors of the fitted parameters. Using PMD 4 shows the lowest errors in most cases. For this wavelength window, the effect of the vertical extension of the FOV and the (wavelength dependent) limb darkening compensate such that the shape assumed by Eq. (1) is best matched. In the following parts, we will only refer to the fits using raw $40 \mathrm{~Hz}$ data of PMD 4 .

As discussed in Sect. 3.1, the validity of the assumptions behind Eq. (1) decreases with the distance of the measurement from the center of the solar disk. Therefore, only the points near the center should be used for the fit. On the other hand, more points means more information is available within the fit. We optimised the number of used points by looking at the errors of the fit. The amount of points is defined by the lowest relative intensity, which should be used for the fit. The smallest errors are reached with a lower limit of the intensities of 0.5 of the maximum intensity.

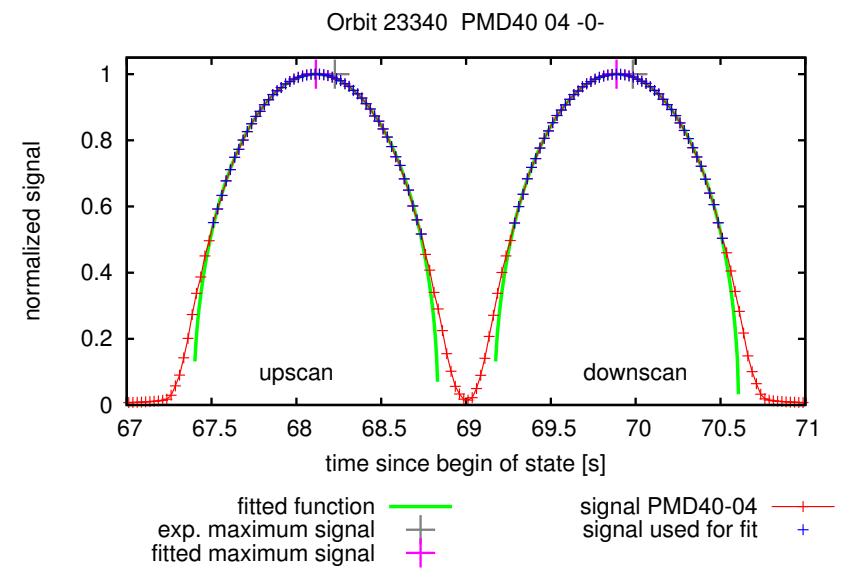

Fig. 5. One up-scan and one down-scan over the solar disk from orbit 23340 (17 August 2006, first scans above $100 \mathrm{~km}$ ). In red the measured intensities are plotted, the blue crosses mark the used measurements. The green lines show the fitted functions. The large purple cross marks the fitted maximum, the large gray cross the position, where the Sun is expected at that time.

\subsection{An example scan}

Figure 5 illustrates the fit for one up-scan and one down-scan. The red crosses show all intensities measured by PMD 4 for a scan sequence at about $120 \mathrm{~km}$ of orbit 23340 (normalized to the maximum signal). The blue crosses denote the measurements actually used for the fits (the normalized signal is larger than 0.5). The green lines are the fitted functions $I(t)$. The large purple cross marks the fitted maximum intensity, i.e. $I\left(t_{\mathrm{cen}}\right) \cdot t_{\mathrm{cen}}$ is the quantity we are interested in: the time when SCIAMACHY points to the center of the solar disk. The large gray cross indicates the time, when the maximum intensity was expected according to the calculated position of the Sun. The distance between the large crosses is proportional to the observed elevation mispointing.

\subsection{Sequence of elevation angle offsets}

At lower tangent heights, the apparent solar position is different from the geometrical solar position due to refraction in Earth's atmosphere (Fig. 2). Using the CFI library, we investigated the effect of the refraction on the apparent solar position for radiation with a wavelength of $650 \mathrm{~nm}$ (the center wavelength of PMD 4). At $40 \mathrm{~km}$ altitude, the solar disk is still shifted by $\sim 200 \mathrm{~m}$, at $60 \mathrm{~km}$ a shift of about $15 \mathrm{~m}$ remains. At $75 \mathrm{~km}$, the difference drops below $1.7 \mathrm{~m}$, which we consider to be negligible for our calculations.

36 scans over the solar disk with the geometrical solar center above $75 \mathrm{~km}$ tangent height are available in state 49 , so for each scan an EAO can be determined. In Fig. 6, the retrieved EAOs for three occultation states are plotted. As can be seen, the individual offsets are time dependent. Assuming a linear dependency, we can fit the equation 


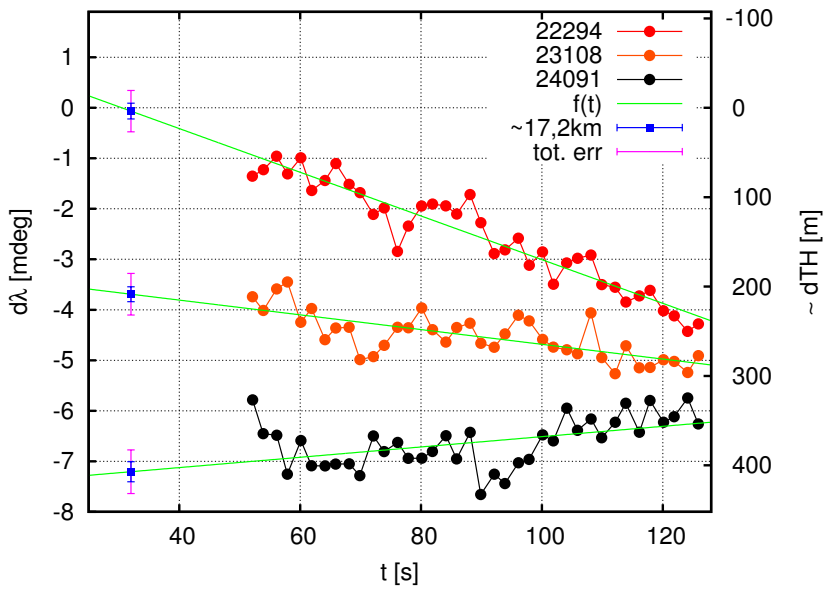

Fig. 6. Fitted EAOs $d \lambda$ versus measurement time $t$ for the occultation states of the orbits 22294 (5 June 2006), 23108 (1 August 2006), and 24091 (9 October 2006). Added are the linear fits $(f(t))$ through these values. The blue square marks the extrapolated EAO at $17.2 \mathrm{~km}$ with the fit error. The violet error bar indicate the total error of the EAO at this altitude (see Sect. 3.5).

$d \lambda(t)=a+b t$

where $d \lambda(t)$ is the EAO at time $t$, and $a$ and $b$ are the fitted intercept and slope. The slope $b$ will be further discussed in Sect. 5.4. For most scientific applications, the EAOs at altitudes in the lower atmosphere are relevant. A linear extrapolation to $32 \mathrm{~s}$ using Eq. (3) is performed. At this time, the (expected) tangent altitude of the geometric Sun (i.e. neglecting refraction effects of Earth's atmosphere) is $17.2 \mathrm{~km}$. This is the case for all solar occultation measurement, because the measurement sequences are planned around this point. This extrapolated EAO is used as the relevant EAO of an occultation state in Sect. 5 (if not stated otherwise).

The remaining error on this EAO (and therefore also on the elevation angle around $17.2 \mathrm{~km}$ ) has two sources. First, we have the error from the linear extrapolation. The linear regression gives the standard errors for the parameters $a$ and $b$ in Eq. (3). These errors are propagated to the error of the EAO at $17.2 \mathrm{~km}$. Second, we consider the uncertainty of the individual scans. As seen in Fig. 6, the EAO for the individual scans scatter around the fitted line. This uncertainty for the individual scan will also disturb a retrieval of an atmospheric parameter at the lower altitude. It can be calculated as standard deviation of the individual EAOs from the fitted line. This error represents the limited precision of the scanner unit. We use here the mean standard deviation over the whole time series as error. The total error on the EAO is the root of the squared sum of both errors. In Fig. 6, the fit error is marked with blue, the total error with violet error bars.

Assigning the correct time to the measurement is essential. If a timing offset occurs, the assigned elevation angle would change in different directions for up- and down-scan, resulting in a zigzag pattern in the sequence of EAOs. A feature of the PMDs is the so-called "analogue delay". That is the time, the read-out electronics consume during transferring the measurement information into binary units. With the default analogue offset for PMD 4 of $13.3 \mathrm{~ms}$, the elevation angle offset versus time (as in Fig. 6) shows a small, but clear zigzag pattern from the alternating up- and down-scans. Such a zigzag pattern increases also the error of the linear fit. We repeated the calculations for the whole dataset with adding 0.00 to $-1.00 \mathrm{~ms}$ in $0.25 \mathrm{~ms}$ steps to the analogue offset and found a minimum of the mean fit error for $-0.75 \mathrm{~ms}$. Therefore, we use $13.225 \mathrm{~ms}$ as analogue offset throughout this paper.

\section{Excluded measurements}

Further on in the paper, we will analyze time series of the observed EAOs. A few measurements have to be excluded before.

\subsection{Solar eclipses}

Over the years, SCIAMACHY observed five partial (at least from SCIAMACHY's point of view) solar eclipses during the solar occultation measurements: 14 October 2004, 29 March 2006, 1 August 2008, 4 January 2011, and 1 June 2011. The first one even affected two subsequent occultation measurements. Figure 7 shows the effect on the observed intensity and the coverage of the solar disk. The relative positions and diameters are calculated with the CFI library (Sect. 3.2).

The observed shapes of the intensity functions are not inline with the assumptions in Sect. 3.1 and the solar eclipse measurements are excluded from our calculations.

\subsection{Outliers}

For about 480 orbits (out of more than 39000 with state 49), the sequence of EAOs in a state shows outliers in the fitted EAOs from the individual scans over the solar disk. Figure 8 shows two examples with $10 \mathrm{mdeg}$ and $8 \mathrm{mdeg}$ different offsets for one scan to the other scans of the same occultation sequence. However, the fit errors for the outliers in fitting Eq. (1) are not larger than that for the other scans. This means that not a single readout failed, but the whole scan over the disk is misplaced by the observed additional angle. We also checked the intensities to verify this conclusion.

We have no explanation for this behavior, it seems to be an occasional problem of the scanner unit. The misplaced scan randomly appears in the scan sequence, as well as such sequences randomly appear over SCIAMACHYs lifetime. In a few cases, even more than one scan over the solar disk is displaced. The maximum additional EAO is about $10 \mathrm{mdeg}$. Please note that this is still well within the pointing requirements and is only slightly larger than 


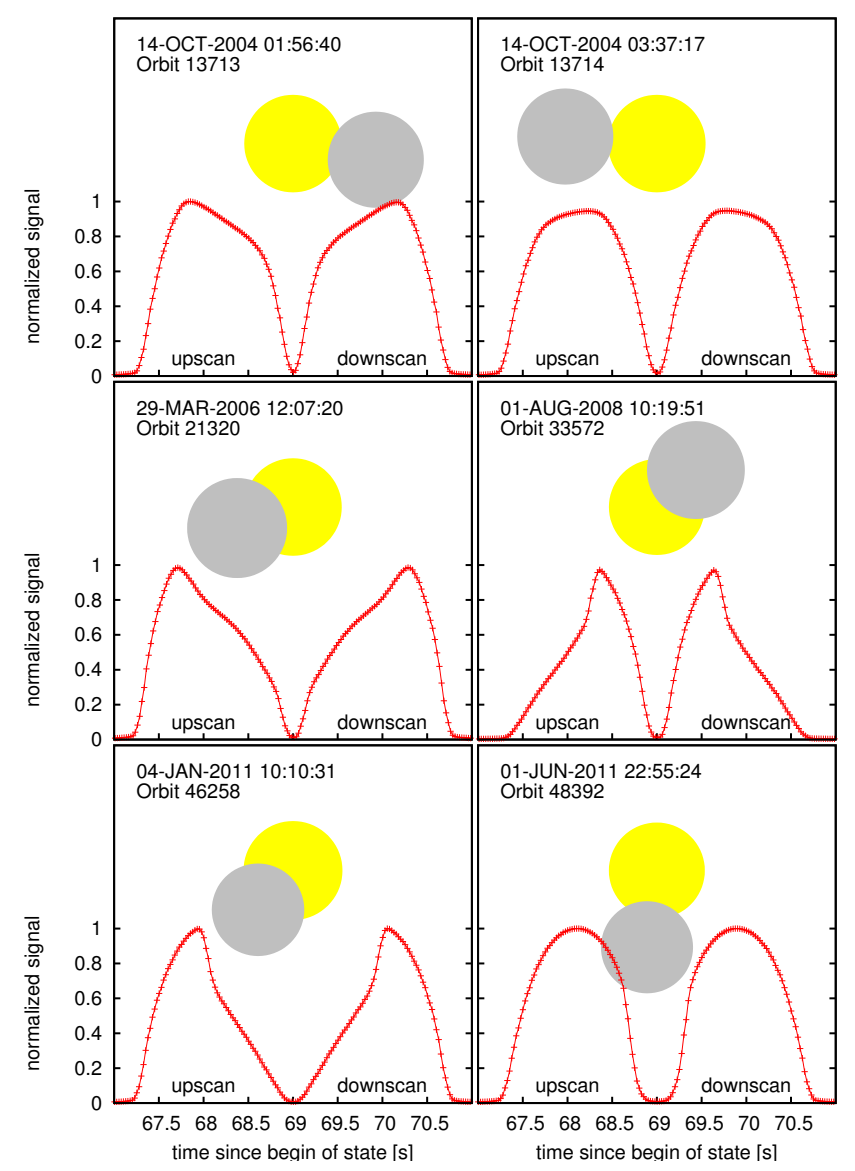

Fig. 7. Observation of solar eclipses during SCIAMACHY's solar occultation measurements. Plotted is the normalized signal of PMD 4 of the first up- and down-scan above $100 \mathrm{~km}$ of the affected orbit. The first two plots are subsequent orbits, observing begin and end of the solar eclipse of 14 October 2004. The coverage of the Sun by the Moon is pictured by the yellow and gray circle. The diameters of Sun and Moon and the relative elevation and azimuth angles are consistently scaled.

SCIAMACHY's error budget of the relative pointing error in elevation ( $8 \mathrm{mdeg})$.

The affected orbits were identified by looking at the errors of the (linear extrapolated) EAO at $32 \mathrm{~s}$, which is enlarged by an outlier in the fitted data (compare the errors in Figs. 6 and 8$)$. With the chosen limit $(0.6 \mathrm{mdeg})$ on the fit error, additional elevation angle offsets larger than 2.5 mdeg are classified as outlier. The orbits with outliers are also excluded from the time series discussed further on.

In the occultation measurement in orbit 47991 (5 May 2011), the scans cover only parts of the solar disk, caused by an in-plane manoeuvre just at the time of the measurement. The incomplete scans are not suitable for our method, therefore this orbit is also excluded.

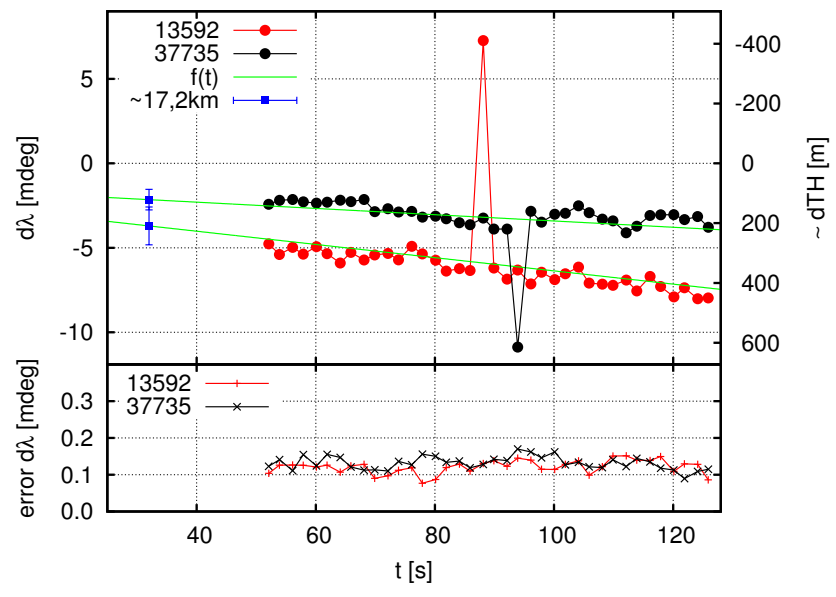

Fig. 8. The top panel shows the fitted EAOs versus measurement time as in Fig. 6, but for the occultation states for the orbits 13592 (5 October 2004) and 37735 (19 May 2009). Both show an outlier in the individual scans. The lower panel shows the error on $t_{\mathrm{cen}}$ for the individual fits (converted to an error on the EAO using the scan velocity).

\section{Time series}

In this section, we analyze time series of the derived EAO and related quantities. Figure 9 comprises all time series plots, which will be discussed in the following sections. For three of the plots, a two month period is shown in detail in Fig. 10.

\subsection{Elevation angle offset history}

Figure 9a shows the full time series of the retrieved EAOs. The maximum observed EAO are from -0.08 to $+0.12 \mathrm{deg}$ (corresponding to about +4.5 to $-6.7 \mathrm{~km}$ offset in tangent height). Most EAOs are from -10 to $+2 \mathrm{mdeg}(+560$ to $-120 \mathrm{~m}$ ), which will be analyzed in detail in Sect. 5.2.

In Fig. 9a, all known platform events with possible impact on the pointing knowledge are marked in blue. Orbit control manoeuvres (OCMs) are the (more or less) regular activities to maintain Envisat's orbit. Most of them have no impact on the finally calculated solar elevation angles. For a few events, the subsequent orbits show larger solar elevation angle deviations: 21 July 2009, 29 September 2009, 8 December 2009, 16 February 2010, 27 April 2010, 6 July 2010, and 1 March 2012. The Envisat attitude anomalies always lead to larger deviations in the elevation angles; the dates are 21 June 2004, 15 February 2009, 11 January 2010, and 26 May 2010. Collision avoidance manoeuvres are necessary, if space debris crosses the planned Envisat orbit. During these manoeuvres, no measurements are performed. The two major events of this type (1 September 2004 and 4 November 2009) did not lead to an enlarged mispointing in the subsequent orbits.

In the early phase of the mission, a large seasonal cycle with an amplitude of about $15 \mathrm{mdeg}(850 \mathrm{~m})$ and 


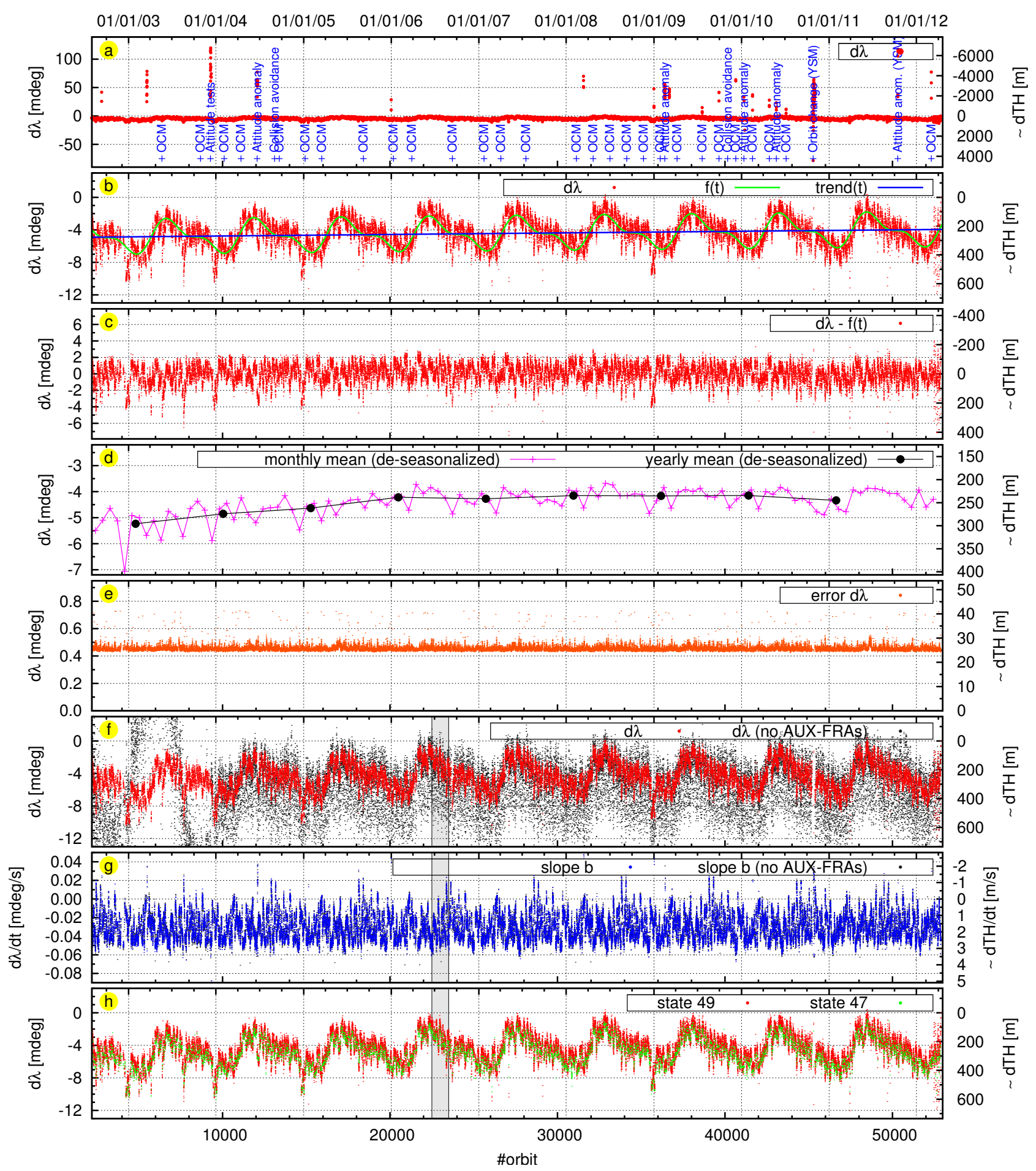

Fig. 9. Time series of fitted EAO, extrapolated to the relevant altitude $\sim 17.2 \mathrm{~km}$. Zooms of the gray bars in (e), (f), (h) are shown in Fig. 10 . (a) Full range of observed EAO. The blue crosses and text mark known instrument anomalies with possible impact on pointing. (b) Zoom of (a) to the main range of observed EAO. Added are the fitted function $f(t)$ according Eq. (4) and the linear term of $f(t)$ (labeled trend $(t)$ ). (c) The remaining day to day variation, if $f(t)$ is subtracted from the EAO. (d) Monthly and yearly means of the EAO with the sinusoidal term of Eq. (4) subtracted. (e) Total error of the EAO. (f) The EAO with and without (gray dots) using the AUX-FRAs. (g) The slope $b$ of the EAO with and without (gray dots) using the AUX-FRAs. (h) EAO derived from state 47, compared to the usual ones from state 49. 


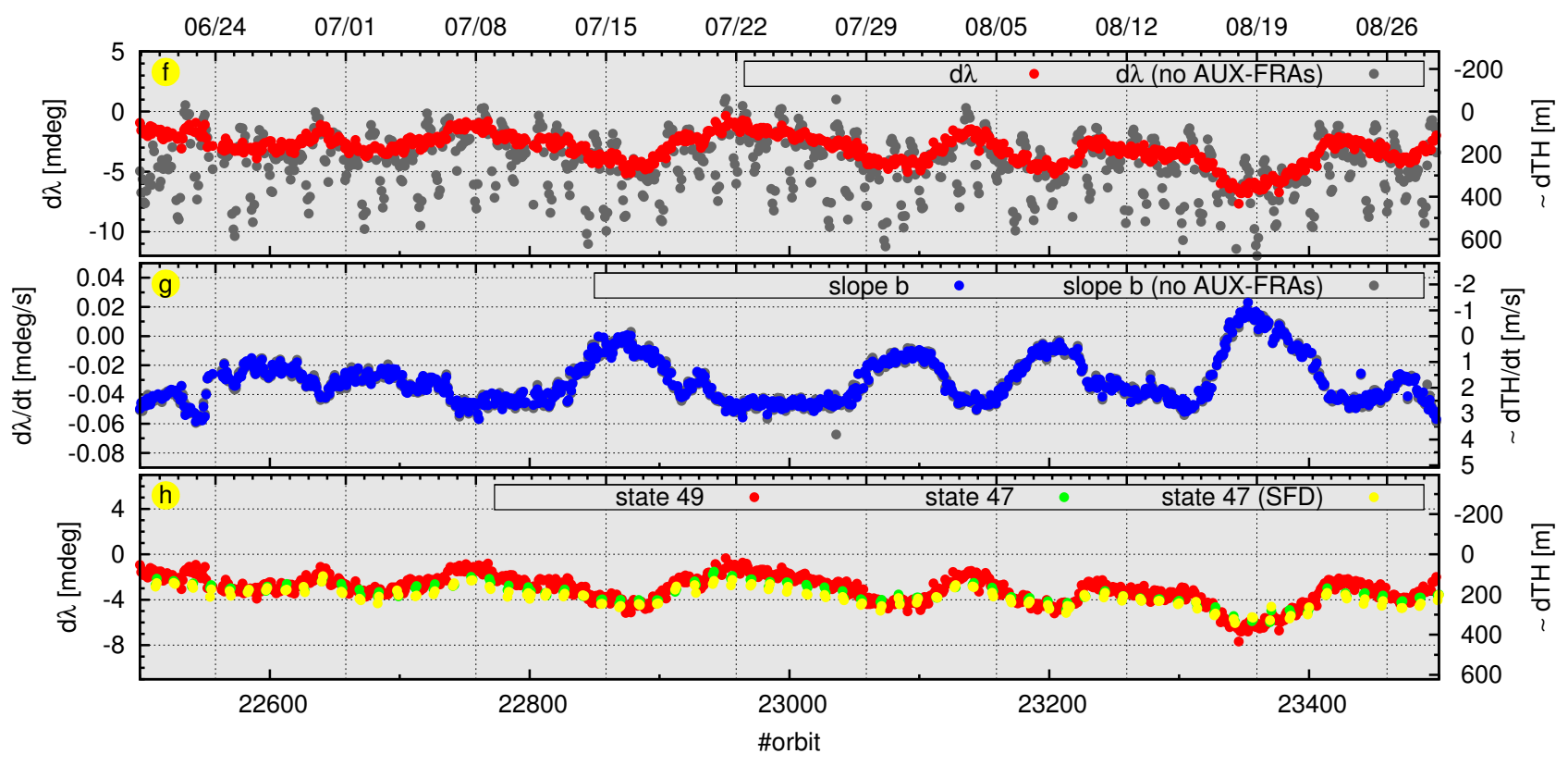

Fig. 10. Zoom of Fig. 9 (f-h) for the time range June to August 2006.

discontinuities in the pitch control where observed. In December 2003, Envisat's on-board software responsible for the attitude control along an orbit, was updated (Bargellini et al., 2005). For this update, the platform was in yaw steering mode with limited pointing accuracy for three days (marked as Attitude tests in Fig. 9, 9-12 December 2003).

At the end of 2010, the Envisat orbit was lowered by $17 \mathrm{~km}$. During the main manoeuvres, Envisat was in YSM (27 October-2 November 2010), marked as Orbit change. Four additional attitude anomalies are known, all of them had impact for a few orbits.

A few larger mispointing events without known reason are visible in the plot, for example in March 2003 and March 2008.

\subsection{Trend analysis}

For the trend analysis, we excluded the mispointing anomalies, i.e. all EAO below $-12 \mathrm{mdeg}$ and above $2 \mathrm{mdeg}$ are not used. The remaining pattern shows a stable seasonal cycle (Fig. 9b). To investigate long-term trends in the observed EAO, we fitted the function

$f(t)=A_{1} \sin \left(2 \pi\left(t+B_{1}\right)\right)+A_{2} \sin \left(4 \pi\left(t+B_{2}\right)\right)+C+D t$

to the time series of the EAO. The time coordinate $t$ is used in the unit years with $t=0.0 \mathrm{yr}$ corresponding to orbit 0 . The sinusoidal terms correspond to an annual and a semi-annual cycle. $A_{1 / 2}$ are the amplitudes, $B_{1 / 2}$ are the phases of the cycles. Additionally, a constant offset $C$ and an overall linear trend with slope $D$ is fitted. The fitted parameters of Eq. (4) are summarized in Table 2. The combined amplitude of the sinusoidal terms, i.e. the amplitude
Table 2. Fitted parameters and its errors according to Eq. (4) of the trend analysis for the elevation angle offset (EAO) and the azimuth angle offset (AAO, see Sect. 8).

\begin{tabular}{lrr}
\hline & EAO & AAO \\
\hline$A_{1}[\mathrm{mdeg}]$ & $1.701 \pm 0.008$ & $2.260 \pm 0.015$ \\
$B_{1}[\mathrm{yr}]$ & $0.906 \pm 0.001$ & $0.645 \pm 0.001$ \\
$A_{2}[\mathrm{mdeg}]$ & $-0.827 \pm 0.008$ & $0.205 \pm 0.015$ \\
$B_{2}[\mathrm{yr}]$ & $1.647 \pm 0.001$ & $1.507 \pm 0.006$ \\
$C[\mathrm{mdeg}]$ & $-4.943 \pm 0.013$ & $88.998 \pm 0.023$ \\
$D\left[\mathrm{mdeg} \mathrm{yr}^{-1}\right]$ & $0.098 \pm 0.002$ & $-0.241 \pm 0.004$ \\
\hline
\end{tabular}

of the seasonal variation is $2.19 \mathrm{mdeg}$, corresponding to $\sim 124 \mathrm{~m}$ in tangent height. The mean EAO of the observed period is $-4.41 \mathrm{mdeg}(\sim 249 \mathrm{~m})$, the overall linear trend is $0.098 \mathrm{mdeg} \mathrm{yr}^{-1}\left(\sim-5.5 \mathrm{~m} \mathrm{yr}^{-1}\right)$. Subtraction of $f(t)$ from the data shows the short-term variation of the EAOs (Fig. 9c). These are in the order of \pm 3 mdeg within ten days. Please note that these short time variations are not random, subsequent orbits differ in the order of only $0.2 \mathrm{mdeg}$. The short time variations are visible in Fig. 10f.

Subtracting only the sinusoidal terms in Eq. (4) from the EAOs, we de-seasonalize the data to investigate long-term trends in more detail. Figure 9d shows monthly and yearly (August-July) means. The amplitude of the remaining pattern in the monthly means is a factor of 4 smaller than the main variation, indicating that most of seasonal variation is captured by the annual and semi-annual cycle. The yearly means give a more precise picture of the trend: the mean elevation angle offset is reduced from $-5.1 \mathrm{mdeg}$ in the first 
year to $-4.2 \mathrm{mdeg}$ in 2006 , further on the mean elevation mispointing remains stable. This corresponds to a mean tangent height offset of about $241 \mathrm{~m}$ since 2006 .

\subsection{Errors of the elevation angle offset}

The total error on the EAO is plotted in Fig. 9e. The mean total error is $0.46 \mathrm{mdeg}(26 \mathrm{~m})$, most errors are below $0.6 \mathrm{mdeg}$. The mean uncertainty of the individual scans is $0.32 \mathrm{mdeg}$ $(24 \mathrm{~m})$. As a reminder, $0.6 \mathrm{mdeg}$ has been chosen as the fit error limit for the detection of sequences with one or more outliers in the individual EAO (Sect. 4.2), corresponding to 0.63 mdeg total error.

\subsection{Slope of the elevation angle offset}

Here we investigate the slopes $b$ of the linear fit according to Eq. (3). The time series of $b$ is plotted in Fig. $9 \mathrm{~g} . b$ is in the range of -0.06 to $+0.02 \mathrm{mdeg} \mathrm{s}^{-1}$, without a clear seasonal cycle.

In Sect. 3.2, we introduced the AUX-FRAs. Figure 9f shows the EAO with and without (red/gray dots) applying the AUX-FRAs for the calculated solar elevation angle. The AUX-FRAs improve the knowledge about the attitude of satellite and therefore about the solar elevation angle. Until the on-board software update in December 2003, the in-flight attitude control leads to larger mispointings up to 20 mdeg. The AUX-FRAs are therefore very important until that date for reliable attitude information. The slope remains unchanged by the application of the AUX-FRAs (the gray dots are just behind the blue ones).

The zoom into June-August 2006 in Fig. 10f and g shows a clear anti-correlation of the short-term variation of EAO and the slope $b$, when the AUX-FRAs are used. The correlation coefficient between these two quantities is -0.73 , if the fitted function $f(t)$ according to Eq. (4) is subtracted from the EAO. It seems that the information content of the AUXFRAs is somehow connected with the observed slope. This observation might help to further improve the AUX-FRAs.

\section{The shortened occultation measurement}

Up to now, we discussed only the solar occultation measurements of state 49 with 36 scans over the solar disk above $75 \mathrm{~km}$. For state 47 , the last up- and down scan sequence crosses the center of the solar disk at about 96 and $104 \mathrm{~km}$ tangent height, respectively. Afterwards, the elevation mirror is moved to the pre-calculated center of the solar disk, and the Sun follower device (SFD) takes over the control also for the elevation mirror. Two seconds of pointing to the Sun completes the state.

The last six scans over the solar disk are used as before to fit the measured solar center. The mirror angles controlled by the SFD are directly compared to the solar elevation angles according to the CFIs. The 80 readouts in $2 \mathrm{~s}$ of pointing are

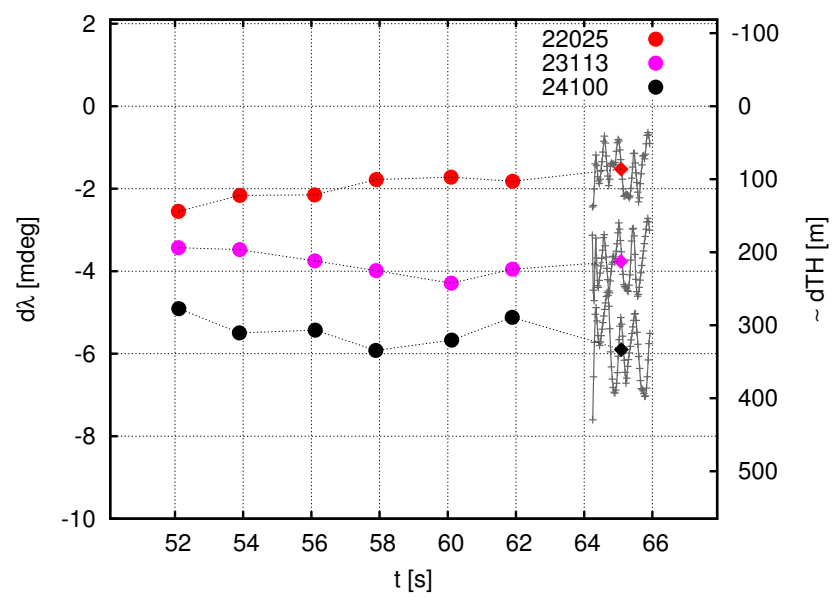

Fig. 11. Examples for the EAOs retrieved for state 47 (orbits 22025 , 23113 , and 24100). The points at 60 and $62 \mathrm{~s}$ are the fits from the last up- and down-scan. The crosses are the EAOs of the individual readouts, when the SFD directs the mirrors. The oscillations of the control loop are clearly visible. The point at $65 \mathrm{~s}$ is the mean of the individual SFD values.

available. The first 12 readouts are skipped, because this time is needed to move from the predicted position of the Sun to the actual one. For the remaining 68 readouts, the EAOs are determined and the arithmetic mean of these values is calculated.

Figure 11 shows three examples for the EAOs from state 47. Shown are the offsets from the last six scans and the mean offset from the pointing phase. All seven points give consistent results, especially the SFD point is inline with the general approach in this paper. The determination of the slope of the EAO (according to Eq. 3) is not possible, as the six scans above $75 \mathrm{~km}$ are not enough for a reliable fit.

Also shown are the EAOs of the individual readouts of the pointing phase. The oscillations around the mean elevation offset directly shows the control loop between the scanner unit and the SFD. The amplitude of the oscillations is about 1 mdeg.

In Fig. 9h, the EAOs from state 47 are compared to the results from state 49 . The overall agreement is very good for the complete time series. Figure $10 \mathrm{~h}$ shows a very good agreement also on an orbital basis, from the fit over the solar disk as well as from using the SFD. Especially the consistency with the SFD results indicates the validity of our approach. Please note: the state 49 EAO is extrapolated to $17.2 \mathrm{~km}$, whereas the state $47 \mathrm{EAO}$ is valid for around $100 \mathrm{~km}$ tangent altitude. For negative values of $b$, the state $49 \mathrm{EAO}$ is slightly higher, vice versa for positive $b$.

\section{Tangent heights for retrievals}

For scientific retrievals, the elevation angle itself is not relevant. Instead, the tangent heights of the line of sights are 
necessary, which define the altitudes of the measured spectra and therefore the altitude grid of a retrieved profile. Therefore, the tangent heights have to be recalculated using the improved elevation angles. We derive the parameters $a$ and $b$ of Eq. (3) and have to correct the given elevation $\lambda$ (determined by the position of the elevation mirror) to get the improved elevation angle $\lambda^{\prime}$ :

$\lambda^{\prime}(t)=\lambda(t)-(a+b t)$.

$\lambda^{\prime}$ is then used as elevation angle to re-calculate the geolocation of the measurement including the tangent heights.

For correct tangent heights in the solar occultation, the readout characteristics of SCIAMACHY's science channels have to be taken into account. For the channels 1-5, standard photodiodes with a sequential readout are used. The time stamp is given for the begin of the readout (in the Level $1 \mathrm{~b}$ product used for this study). The readout of all 1024 pixel takes $28.771 \mathrm{~ms}$. If the relevant information comes from pixel number $n$ of the channel, than the geolocation has to be calculated for $t=t_{\mathrm{L} 1 \mathrm{~b}}+(n / 1024) \cdot 28.771 \mathrm{~ms}$. The channels 6,7 and 8 use InGaAs detectors, which are readout in parallel. For solar occultation, channels 6 and 7 have integration times of $31.25 \mathrm{~ms}$, which is half of the minimum readout cycle time of $62.5 \mathrm{~ms}$. This means, the first $31.25 \mathrm{~ms}$ of the measurement are thrown away and not used. The mid of the integration time (which should be used for the mean pointing) is therefore shifted by half of this time: $t=t_{\mathrm{L} 1 \mathrm{~b}}+15.625 \mathrm{~ms}$.

Recognizing the derived function for the EAO and the readout characteristics leads to a very precise tangent height information for the solar occultation measurements. Within the retrievals, the vertical gradient of the atmosphere in the FOV and the elevation change during the integration of the signal have to be taken into account.

SCIAMACHY's limb measurement have the same requirements for the pointing knowledge. However, the EAO derived from solar occultation cannot be used directly. The viewing direction in limb is in the flight direction of Envisat, whereas the occultation viewing direction is in azimuth about 30 deg left of the ground track. Because we cannot determine, which axes of the platform are involved in the mispointing, the limb elevation offset might be different. The solar occultation measurement are all in northern mid to high latitudes (see Fig. 1), whereas the limb measurements are globally distributed. If we have an orbital pattern in the EAO, we cannot detect this from the solar occultation measurement alone.

The most recent results of the TRUE method for limb measurements in Gottwald et al. (2010) show also a seasonal variation. However, the phase is shifted and the amplitude is smaller compared to the results shown here. Therefore, we think that the tangent height offset indeed has an (currently unknown) orbital component.

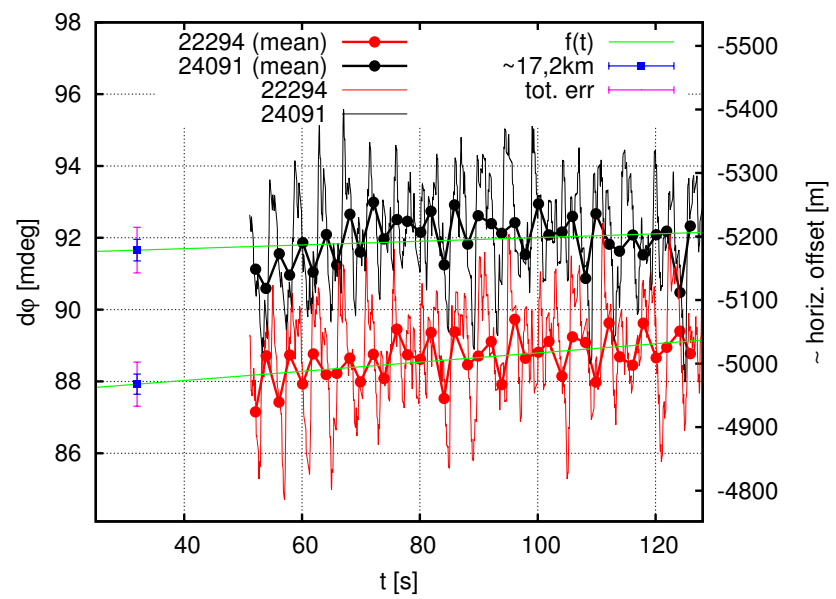

Fig. 12. Fitted AAOs versus measurement time for the occultation state for two of the orbits already used in Fig. 6: 22022 (17 May 2006), and 24091 (9 October 2006). The thin lines indicates the individual readouts, the points are the mean over one scan. Added are the linear fits $(f(t))$ through these values. The blue square marks the extrapolated EAO at $17.2 \mathrm{~km}$ with the fit error. The violet error bar indicates the total error of the EAO at this altitude (see Sect. 3.5).

\section{Azimuth angle}

A high precision azimuth angle is less important for scientific retrievals, because no horizontal gradients on small scales are expected in the upper atmosphere. In azimuth direction, the SFD takes over control, when the geometric Sun reaches an altitude of $17.2 \mathrm{~km}$. Therefore, we can directly compare the azimuth angles of the mirrors to the azimuth calculated by the CFIs to estimate the azimuth angle offset (AAO), as we did for the elevation during the pointing phase of state 47. Here, we calculated the mean AAO for one scan over the solar disk and took this as the AAO for this scan. Figure 12 shows two example orbits. The individual readouts indicate the control loop of the SFD. The amplitude is about $2.5 \mathrm{mdeg}$, which is more than twice of the amplitude in elevation (as observed in state 47). The SFD is less accurate in azimuth direction (Schwab, 1999), and for part of the scan in elevation, the Sun is not in the IFOV, i.e. the SFD has no signal to adjust. As for the elevation angle offsets, we determined the AAO for each orbit (with state 49 measurements) as extrapolation to the $17.2 \mathrm{~km}$ altitude and analyzed the time series. Again, we excluded the mispointing events from the time series: AAOs below $78 \mathrm{mdeg}$ and above $97 \mathrm{mdeg}$ are not used. Figure 13b shows the trend analysis for the AAO. As done for the EAO, the function described by Eq. (4) is fitted to the AAO dataset. The fitted parameters are listed in Table 2. The amplitude of the seasonal cycle is $2.26 \mathrm{mdeg}$ (corresponding to $\sim 127 \mathrm{~m}$ in horizontal direction at the tangent point), dominated by the yearly sinusoid. In Fig. 13b it is also visible that the overall seasonal cycle is not matched in 2002 and slightly different 


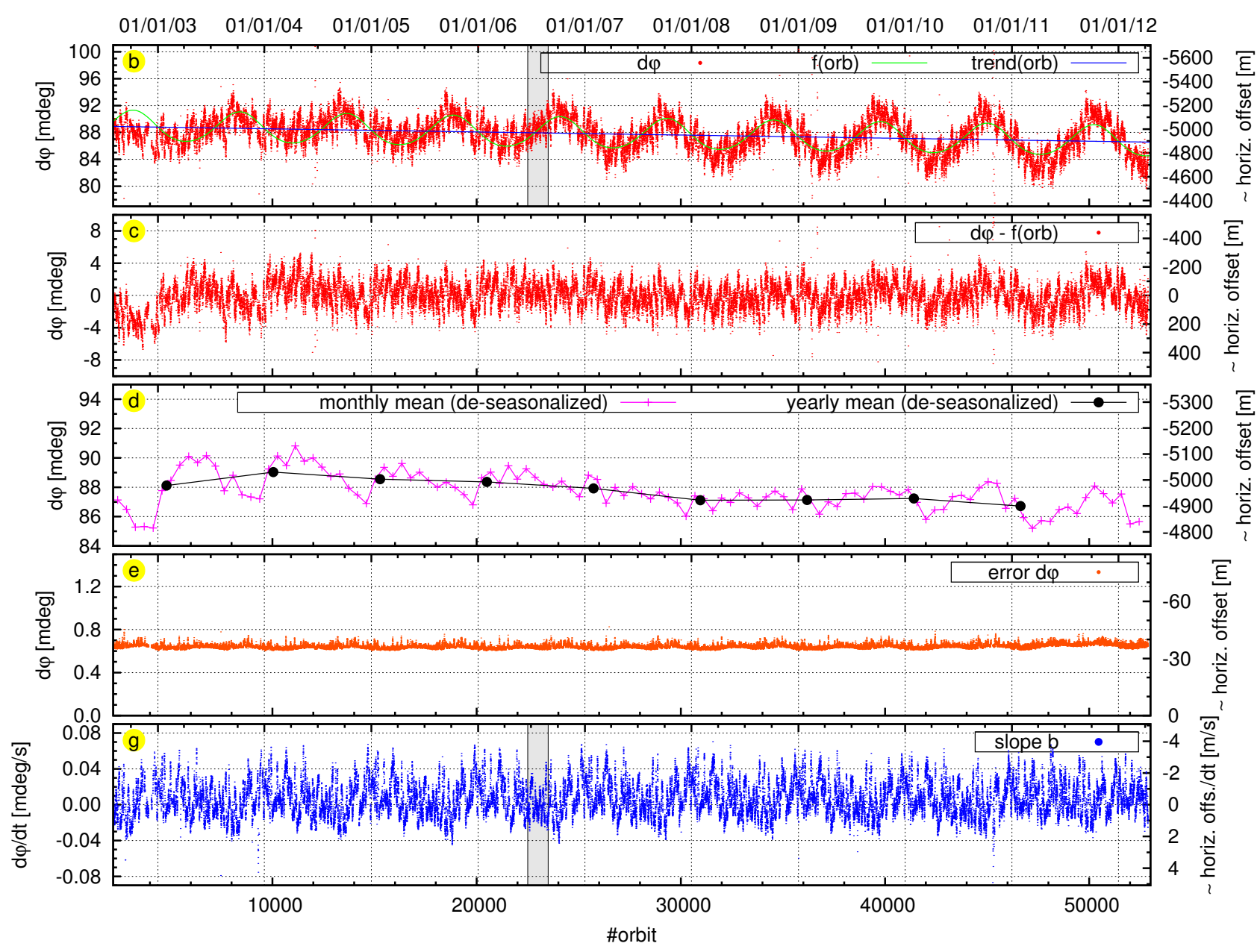

Fig. 13. Time series of the AAO $d \varphi$, derived from the adjustment of the azimuth mirror by the Sun follower device (SFD). The plots corresponds to Fig. 9 (b-e) and (g) for the elevation angle. Zooms of the gray bars in (b) and (g) are shown in Fig. 14.

in 2003. Towards the later years, the amplitude slightly increases with time.

Remarkable is the mean AAO of $87.7 \mathrm{mdeg}$, corresponding to a mean horizontal offset of almost $5 \mathrm{~km}$ for the occultation measurements. The observed overall linear trend is $D=-0.241 \pm 0.004 \mathrm{mdeg} \mathrm{yr}^{-1}$. The overall small trend is confirmed by the monthly and yearly mean in Fig. 13d, which are again calculated with the sinusoidal term subtracted from the data. The errors of the AAO are shown in Fig. 13e. The mean total error is about $0.65 \mathrm{mdeg}(36 \mathrm{~m})$, most errors are below 0.9 mdeg.

The mean scanner unit error (standard deviation of the individual AAOs from the fitted line) is $0.59 \mathrm{mdeg}(33 \mathrm{~m})$. The errors for the azimuth are in general by a factor of 1.4 higher than for the elevation. The errors are slightly higher at the begin and towards the later phase of the mission.

Figure 13g shows the slope of the AAO, corresponding to Fig. $9 \mathrm{~g}$ for the EAO. Both are in the same order of magnitude.

Figure 14 shows the short-term variation of the AAO and the change of the AAO with time for the same period already used in Fig. 10. The AAO data are noisier than the elevation data, the variation from orbit to orbit is in the order of $4 \mathrm{mdeg}$. There are still short-term variations visible, and they are correlated with the slope $b$ of the linear fit through the sequence of solar scans. The correlation coefficient between these two values is -0.61 , if again the fitted function is subtracted from the AAO.

\section{Conclusions}

The sequence of scans over the solar disk during SCIAMACHY's solar occultation measurements have been used to determine the pointing direction in elevation with a mean standard error $(1 \sigma)$ of $0.46 \mathrm{mdeg}$ ( $\sim 26 \mathrm{~m}$ in altitude). This is one order of magnitude better than the original pointing error budget for SCIAMACHY. The mispointing of the instrument shows a clear seasonal cycle with an amplitude of $2.19 \mathrm{mdeg}$ ( $\sim 124 \mathrm{~m}$ in tangent altitude), the overall mean mispointing is $-4.41 \mathrm{mdeg}(\sim 249 \mathrm{~m})$. The values are summarized in 
Table 3. Summary of the statistical quantities of the observed elevation and azimuth angle offsets for SCIAMACHY's solar occultation measurements. The corresponding vertical and horizontal offsets at the tangent point are also listed.

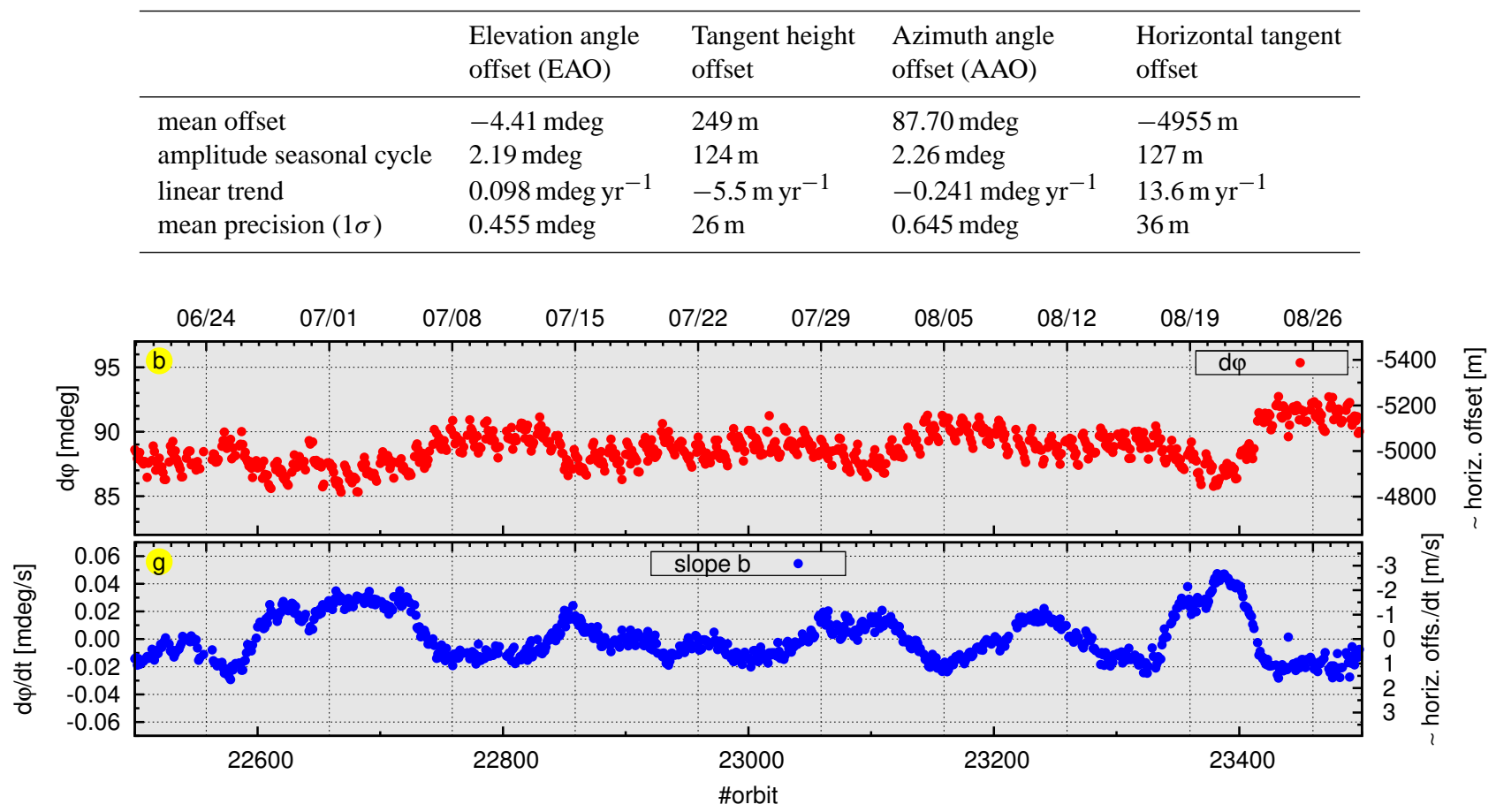

Fig. 14. Zoom of Fig. 13 (b) and (g) with the time range June to August 2006.

Table 3. There is an on-going discussions about a possible trend in SCIAMACHY's (limb) tangent height offsets, which would (partly) explain observed trends in trace gas profiles (Gottwald et al., 2010). From this work, we cannot support such an assumption. There is only a small decrease of the mean tangent height offset in the first four years of the mission of $50 \mathrm{~m}$ in total and a stable pattern since then.

The pointing control by SCIAMACHY's sun follower device allows to determine the azimuth pointing direction with a standard error of $0.65 \mathrm{mdeg}$ ( $\sim 36 \mathrm{~m}$ in horizontal direction). A seasonal cycle with an amplitude of 2.26 mdeg ( $\sim 127 \mathrm{~m})$ and a mean offset of $87.7 \mathrm{mdeg}(\sim 5.0 \mathrm{~km})$ is observed for the azimuth angle offset of the instrument.

A detailed analysis of the observed pattern is necessary. Especially the astonishingly strong correlation between the time dependence of the angle offsets and the offset itself, and the small offset changes from orbit to orbit might in the future help to understand the reasons for these pattern and further improve the attitude knowledge for Envisat.

For the occultation measurements, we are now in the position to derive profiles of atmospheric parameters with further improved altitude knowledge compared to previous investigations (Meyer et al., 2005; Bramstedt et al., 2007; Noël et al., 2010, 2011). The presented method can also be used for other instruments scanning over the full solar disk, for example the SAGE III instrument followed a similar strategy in their solar occultation measurements (Cunnold and McCormick, 2002).

Acknowledgements. SCIAMACHY is a national contribution to the ESA Envisat project, funded by Germany, The Netherlands, and Belgium. SCIAMACHY data have been provided by ESA. This work has been funded by DLR Space Agency (Germany) within grant 50EE1105, and by the University of Bremen.

Edited by: J.-P. Pommereau

\section{References}

Alvarez, L.: Envisat-1 Mission CFI Software Mission Conventions Document, Tech. rep., ESA, GMV, S.A., Madrid, PO-IS-ESAGS-0561, available at: http://eop-cfi.esa.int/CFI/cfi_software. html (last access: March 2012), 1997.

Bargellini, P., Matatoros, M. G., Ventimiglia, L., and Suen, D.: ENVISAT Attitude and Orbit Control In-Orbit Performance: An Operational View, in: Proceedings of 6th International ESA Conference on Guidance, Navigation and Control Systems, ESASP 606, ESA Publications Division, Loutraki, Greece, 2005.

Bovensmann, H., Burrows, J. P., Buchwitz, M., Frerick, J., Noël, S., Rozanov, V. V., Chance, K. V., and Goede, A. P. H.: SCIAMACHY: Mission Objectives and Measurement Modes, J. Atmos. Sci., 56, 127-150, 1999. 
Bramstedt, K., Amekudzi, L., Bracher, A., Rozanov, A., Bovensmann, H., and Burrows, J. P.: SCIAMACHY solar occultation: Ozone and $\mathrm{NO}_{2}$ profiles from 2002-2006, in: Proceeding of the ERS-Envisat Symposium, ESA-SP 636, ESA Publications Division, Montreux, 2007.

Cunnold, D. M. and McCormick, M. P.: SAGE III Algorithm Theoretical Basis Document (ATBD) Transmission Level 1B Products, Tech. Rep. LaRC 475-00-108, NASA Langley Research Center, 2002.

Giorgini, J. D., Yeomans, D. K., Chamberlin, A. B., Chodas, P. W., Jacobson, R. A., Keesey, M. S., Lieske, J. H., Ostro, S. J., Standish, E. M., and Wimberly, R. N.: JPL's On-Line Solar System Data Service, in: Bulletin of the American Astronomical Society, 28, p. 1158, 1996.

Gottwald, M. and Bovensmann, H. (Eds.): SCIAMACHY - Exploring the Changing Earth's Atmosphere, Springer, Dordrecht, Heidelberg, London, New York, 2011.

Gottwald, M., Krieg, E., von Savigny, C., Noël, S., Bovensmann, H., and Bramstedt, K.: Determination of SCIAMACHY Lineof-Sight Misalignments, Proc. Envisat Symposium, 23-27 April, SP-636, Montreux, Switzerland, 2007.

Gottwald, M., Krieg, E., Lichtenberg, G., Slijkhuis, S., von Savigny, C., Noel, S., Bovensmann, H., and Bramstedt, K.: The Status of the SCIAMACHY Line-of-Sight Pointing Knowledge, Proc. ESA Living Planet Symposium, 28 June-2 July 2010, SP686, Bergen, Norway, 2010.

Hestroffer, D. and Magnan, C.: Wavelength dependency of the Solar limb darkening, Astron. Astrophys., 333, 338-342, 1998.

Kaiser, J., von Savigny, C., Eichmann, K., Noël, S., Bovensmann, H., and Burrows, J.: Satellite Pointing Retrieval from Atmospheric Limb Scattering of Solar UV-B Radiation, Can. J. Phys., 82, 1041-1052, 2004.

Lourakis, M.: levmar: Levenberg-Marquardt nonlinear least squares algorithms in $\mathrm{C} / \mathrm{C}++$, Version 2.5, available at: http://www.ics. forth.gr/ lourakis/levmar (last access: March 2011), 2009.
Meyer, J., Bracher, A., Rozanov, A., Schlesier, A. C., Bovensmann, H., and Burrows, J. P.: Solar occultation with SCIAMACHY: algorithm description and first validation, J. Atmos. Chem. Phys, 5, 1589-1604, 2005.

Muñoz, C. V.: Re-Engineering of Mission Analysis Software for Envisat-1, General Software User Manual, Tech. Rep. Issue 5.9, ESA, DEIMOS Space S.L.U, Madrid, PO-IS-DMS-GS-0556, available at: http://eop-cfi.esa.int/CFI/cfi_software.html (last access: March 2012), 2011.

Neckel, H.: Analytical Reference Functions $F(\lambda)$ for the Sun's Limb Darkening and Its Absolute Continuum Intensities $(\lambda \lambda$ 300 to $1100 \mathrm{~m}$ ), Sol. Phys., 229, 13-33, doi:10.1007/s11207005-4081-z, 2005.

Noël, S., Bramstedt, K., Rozanov, A., Bovensmann, H., and Burrows, J. P.: Water vapour profiles from SCIAMACHY solar occultation measurements derived with an onion peeling approach, Atmos. Meas. Tech., 3, 523-535, doi:10.5194/amt-3-523-2010, 2010.

Noël, S., Bramstedt, K., Rozanov, A., Bovensmann, H., and Burrows, J. P.: Stratospheric methane profiles from SCIAMACHY solar occultation measurements derived with onion peeling DOAS, Atmos. Meas. Tech., 4, 2567-2577, doi:10.5194/amt-42567-2011, 2011.

Schwab, A.: Pointing Error Budget, Tech. Rep. TN-SCIA0000DO/06, Dornier, 1999.

von Savigny, C., Kaiser, J. W., Bovensmann, H., Burrows, J. P., McDermid, I. S., and Leblanc, T.: Spatial and temporal characterization of SCIAMACHY limb pointing errors during the first three years of the mission, Atmos. Chem. Phys., 5, 2593-2602, doi:10.5194/acp-5-2593-2005, 2005.

Yeomans, D., Chamberlin, A., Giorgini, J., Chodas, P., Jacobson, R., Keesey, M., Lieske, J., Ostro, S., Standish, E., and Wimberly, R.: The JPL HORIZONS on-line solar system data and ephemeris computation service, available at: http://ssd.jpl.nasa. gov/?horizons (last access: March 2012), 1996. 